\title{
DESASTRES NATURAIS E INDUZIDOS E O RISCO URBANO
}

\author{
Maria Giovana Parizzi \\ Departamento de Geologia - IGC/UFMG -mgparizzi@msn.com \\ Recebido em 6 de setembro de 2013; aceito em 5 de junho de 2014
}

RESUMO: O artigo apresenta síntese sobre os atuais conceitos e classificações de desastres, risco e sua relação com a vulnerabilidade ambiental e social. Inclui análise sobre os últimos desastres ocorridos no mundo, no Brasil e em Minas Gerais e os impactos decorrentes destes fenômenos. Discute também sobre critérios adotados para a gestão do risco em áreas urbanas, considerando este um processo social complexo que envolve ações de planejamento, intervenção e organização, que devem ser avaliadas e conduzidas de forma contínua.

Palavras-chave: RISCO GEOLÓGICO, GESTÃO, CENTRO URBANOS.

ABSTRACT: NATURAL AND HUMAN-INDUCED DISASTERS AND URBAN RISK. The article presents an overview of current concepts and disaster risk classifications and its correlation with the environmental and social vulnerability. Includes analysis of the recent disasters in the world, in Brazil and in Minas Gerais and the impacts caused by these phenomena. Also discusses about the criteria adopted for risk management in urban areas considering this a complex social process that involves planning, intervention and action organization, which should be evaluated and implemented on an ongoing basis.

Keywords: GEOLOGICAL RISK, MANAGEMENT, URBAN CENTER

\section{INTRODUÇÃO}

Os centros urbanos encontram-se em constante crescimento. A construção das cidades demanda obras que descaracterizam as paisagens naturais, conduzem ao intenso uso e exploração de materiais de construção e industriais, à explotação dos recursos hídricos, à ocupação de áreas de solos férteis para outros fins, etc.

A avaliação dos componentes do meio físico e a compreensão de sua dinâmica são essenciais para o desenvolvimento do processo de urbanização. Conforme Ministério do Meio Ambiente- MMA (2007) é necessário reconhecer que cada pequena porção de território apresenta um conjunto próprio de respostas frente às mudanças ambientais e diferentes formas de uso e ocupação.

Torna-se necessário refletir sobre tais ações e, para tal, citamos o conceito de paisagem de Bertrand (1972, p.1):

"A paisagem não é a simples adição de
elementos geográficos disparatados. É, em
uma determinada porção do espaço, o
resultado da combinação dinâmica,
portanto instável, de elementos físicos,
biológicos e antrópicos que, reagindo
dialeticamente uns sobre os outros, fazem
da paisagem um conjunto único e
indissociável, em perpétua evolução."

A implantação das cidades impõem solicitações ao meio físico das mais variadas formas, alterando sua dinâmica com a criação ou a aceleração de inúmeros processos. Os processos geológicos fazem parte da dinâmica natural do planeta e sua ocorrência independe da presença do homem. Entretanto, cada alteração do ambiente provocada pelas atividades humanas irá afetar o equilíbrio do meio físico. Toda reação ou resposta ambiental caminha para o reestabelecimento do equilíbrio perdido e, nem sempre, isso será favorável à ocupação e à humanidade. A intensificação das atividades humanas pode induzir, acelerar e potencializar os processos geológicos e isso pode provocar acidentes e desastres que afetam diretamente o homem.

\section{CLASSIFICAÇÃO DOS RISCOS}

Risco geológico é definido pela possibilidade de um processo geológico ou fenômeno causar perda de vida, lesões ou outros impactos sobre a saúde, danos materiais, perda de meios de subsistência e de serviços, perturbações sociais e econômicas ou danos ambientais.

De acordo com Cerri \& Amaral (1998), "a ocorrência de um fenômeno geológico pode ou não gerar perdas e danos. No primeiro caso ele é chamado de acidente e no segundo de evento. A susceptibilidade de uma área com relação a determinado fenômeno geológico caracteriza a possibilidade de sua ocorrência (evento), enquanto que risco envolve a possibilidade de que um fenômeno (evento) seja acompanhado de danos e perdas (acidente)". Vulnerabilidade significa o grau de perda de um dado elemento ou grupo de risco dentro de uma área afetada por um processo.

CRED - Centre for Research on the Epidemiology of Disasters (2009) define um desastre como uma situação ou acontecimento que transpõe a capacidade local de superação do problema e, assim, implica a solicitação de assistência externa a um nível nacional ou internacional. Desastre pode ser associado a um evento imprevisto, que ocorre muitas vezes de forma súbita, e que causa grande dano, destruição e sofrimento humano. Os desastres são a convergência dos perigos com 
vulnerabilidades. Como tal, um aumento da vulnerabilidade ambiental, social ou econômica pode significar um aumento da frequência das catástrofes.

Cerri \& Amaral (1998) consideram os riscos ambientais como a classe maior dos riscos, subdividindo-os em classes e subclasses de acordo com a Figura 1.

\subsection{Desastres naturais, induzidos e mistos}

Os desastres podem ser classificados como naturais e humanos ou induzidos de acordo com Marcelino (2008) conforme Tabela 1.

O Ministério da Integração Nacional, em 2003, apresentou a definição de Desastres Mistos como sendo quando as ações e omissões humanas contribuem para intensificar ou agravar fenômenos potencialmente indutores de desastres. Também se caracterizam quando intercorrências de fenômenos naturais adversos, atuando sobre condições ambientais degradadas pelo homem, desencadeiam desastres.
Os desastres mistos são classificados em dois grandes grupos:

- Desastres mistos relacionados com a geodinâmica terrestre externa.

- Desastres mistos relacionados com a geodinâmica terrestre interna.

\section{Desastres naturais pelo mundo e no Brasil}

Os fenômenos naturais que desencadeiam os desastres, como as tempestades, os terremotos e os vulcões existem em diversas partes do globo e podem ocorrer em qualquer continente ou país. Entretanto, algumas regiões e populações são mais afetadas em função da magnitude e frequência dos fenômenos e da vulnerabilidade do sistema social.

De acordo com o escritório das Nações Unidas para a Redução de Desastres, UN-ISDR (2011), o ano de 2011 foi marcado por 302 desastres naturais, que mataram 29.782 pessoas no mundo, mas principalmente na Ásia. A estimativa é que os desastres geraram US\$ 366 bilhões de prejuízos.

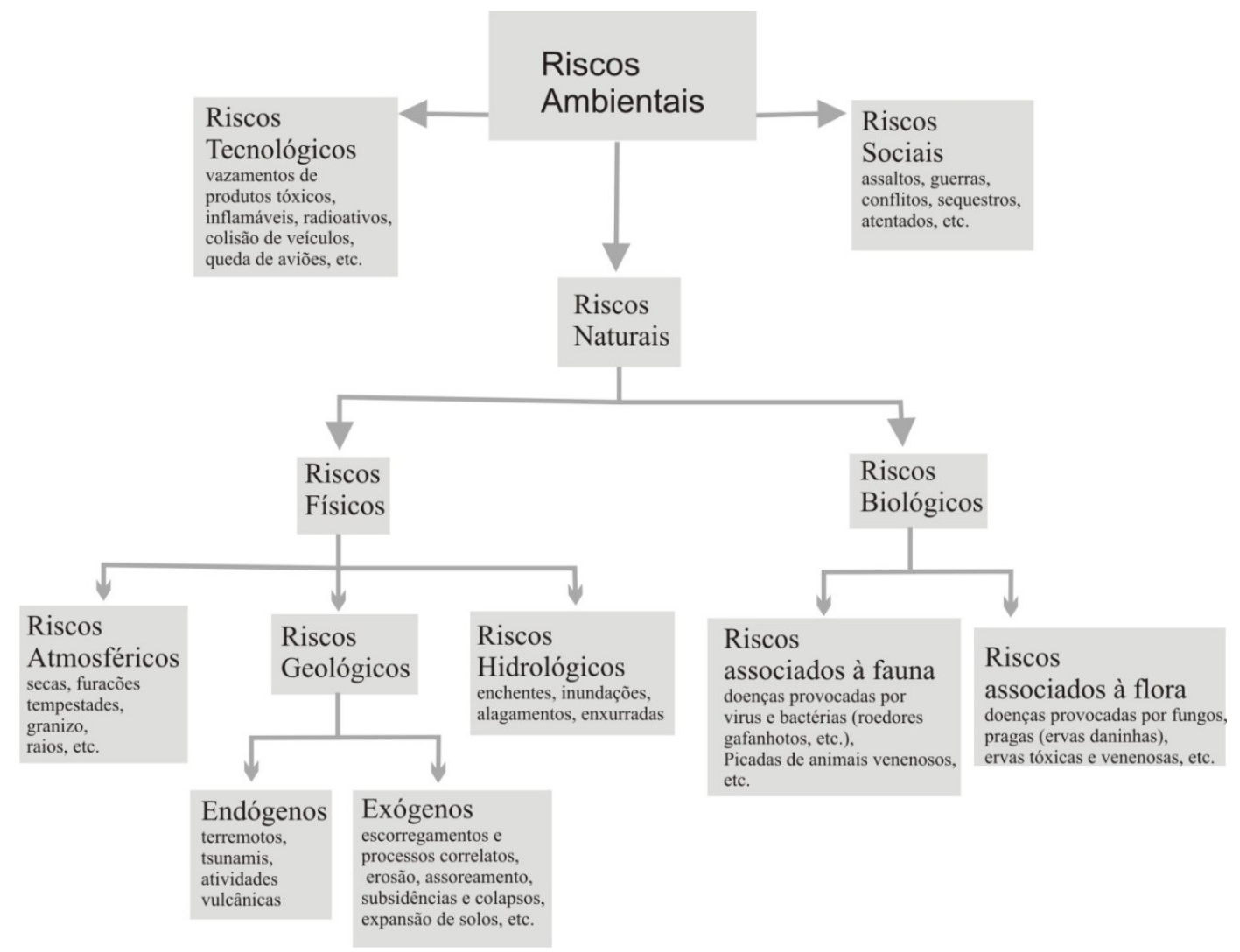

Figura 1 - Classificação de riscos Ambientais (adaptado de Cerri \& Amaral, 1998)

Tabela 1 - Definições dos tipos de desastres, conforme Marcelino (2008)

$\begin{array}{ll}\text { Desastres Naturais } & \begin{array}{l}\text { são aqueles disparados pela intervenção direta de um fenômeno natural de grande intensidade. } \\ \text { Exemplo: fortes chuvas - inundação e escorregamentos, fortes ventos - vendaval, tornado e furacão, } \\ \text { etc. }\end{array} \\ \text { Desastres Humanos } & \begin{array}{l}\text { são aqueles disparados pelas ações ou omissões humanas. Exemplo: acidentes de trânsito, incêndios } \\ \text { industriais, contaminação de rios, rompimento de barragens. }\end{array}\end{array}$


Tabela 2 - Registro de óbitos devido aos intensos terremotos ocorrido em 2010, 2011, 2012 e 2013 (EM-DAT, 2013)

\begin{tabular}{ccccc} 
Ano & Local & Evento & $\begin{array}{c}\text { Magnitude } \\
\text { (Escala Richter) }\end{array}$ & Óbitos \\
\hline 2010 & Haiti & Terremoto & 7 & 220.000 \\
\hline 2011 & Japão & Terremoto/tsunami & 8,9 & 20.000 \\
\hline 2012 & Filipinas & Terremoto & 7,9 & 65 \\
\hline 2013 & China & Terremoto & 5,9 & 70 \\
\hline
\end{tabular}

A maior parte das mortes foi provocada pelos efeitos dos terremotos (Tabela 2). Pelo menos 20.943 pessoas morreram devido às consequências dos sismos. Do total de mortos, 19.846 ocorreram no Japão. Em janeiro de 2010 mais de 220 mil pessoas morreram no Haiti, devido a terremoto. O fenômeno, não ocorria na região há 200 anos. No ano de 2011 também foram registradas inundações no Brasil, terremotos na Nova Zelândia e no Japão seguido por tsunami, além de tempestades acompanhadas por tornados e furacões nos Estados Unidos, alagamentos na Tailândia, tremores de terra na Turquia e tempestades nas Filipinas. A UN-ISDR (United Nations International Strategy for Disaster Reduction) relata ainda que 231 pessoas morreram devido à elevação da temperatura.

Em 2012, 310 catástrofes naturais foram registradas pelo CRED-EM-DAT (CRED-EM-DAT, 2013). Houve perda de 9.930 vidas, e mais de 106 milhões de pessoas afetadas, sendo U\$138 bilhões relacionados a danos econômicos. O maior desastre de 2012 em termos de mortalidade foi o tufão Bopha nas Filipinas, que resultou em 1.901 mortes. Foi o ciclone tropical mais forte registrado na ilha filipina de Mindanao e afetou em torno de 6 milhões de pessoas (CRED-EM-DAT, 2013).

Globalmente, a Ásia, mais uma vez, foi o continente mais afetado pelos desastres naturais em 2012, tanto em termos de ocorrência, pessoas mortas e pessoas afetadas. Por outro lado, $63 \%$ de perdas econômicas ocorreram nas Américas, principalmente devido ao furacão Sandy que atingiu a costa leste dos Estados Unidos em outubro (U\$50 bilhões) e uma seca que afetou $62 \%$ dos Estados Unidos (U\$20 bilhões). Outros desastres notáveis em termos de impacto econômico no referido ano foram dois tremores de terra que atingiram o norte da Itália em maio e que resultou em $U \$ 15,8$ bilhões de danos econômicos (CRED-EM-DAT, 2013).
Embora a maioria dos danos econômicos relacionados a desastres naturais em 2012 tenham ocorrido em nações desenvolvidas, os impactos de desastres em países menos desenvolvidos não devem ser menosprezados. Por exemplo, os danos causados pelo ciclone Evan em Samoa representaram quase $20 \%$ do PIB do país. Da mesma forma, inundações no Paquistão resultaram em perdas iguais a $1,7 \%$ do PIB do país. Apenas abordando a ligação entre a redução da pobreza e a redução do risco de desastres é que as comunidades internacionais poderão reduzir os impactos de catástrofes em países em desenvolvimento (CRED-EM-DAT, 2013).

O Brasil não está fora das estatísticas, registrando mais de 900 mortes causadas pelos impactos das inundações e dos deslizamentos de terras provocados pela chuva no ano de 2011.

De acordo Marcelino (2008), no Brasil, os tipos de desastres mais frequentes são as inundações (representadas pelos tipos graduais e bruscos, com 59\% dos registros), seguidas pelos escorregamentos (14\%). A maioria dos desastres no Brasil (mais de $80 \%$ ) está associada às instabilidades atmosféricas severas, que são responsáveis pelo desencadeamento de inundações, vendavais, tornados, granizos e escorregamentos. Com exceção das inundações graduais, esses fenômenos são súbitos e violentos, responsáveis por grande mortandade e destruição.

De acordo com a Defesa Civil (2013), em um cenário de extensão continental, com cerca de 8,5 milhões km², $7.367 \mathrm{~km}$ de litoral banhado pelo Oceano Atlântico e 182 milhões de habitantes, o Brasil apresenta características regionais de desastres, onde os desastres naturais mais prevalentes podem ser observados conforme a Tabela 3.

Tabela 3 - Distribuição de tipos de desastres por região Brasileira (fonte: Defesa Civil,2013)

$\begin{array}{ll}\text { REGIÃO NORTE } & \text { INCÊNDIOS FLORESTAIS E } \\ \text { INUNDAÇÕES }\end{array}$

REGIÃO NORDESTE SECAS E INUNDAÇÕES

REGIÃO CENTRO-OESTE INCÊNDIOS FLORESTAIS

$\begin{array}{ll}\text { REGIÃO SUDESTE } & \text { DESLIZAMENTOS E INUNDAÇÕES } \\ \text { REGIÃO SUL } & \text { INUNDAÇÕES, VENDAVAIS E } \\ & \text { GRANIZO }\end{array}$


No ano de 2011, os desastres naturais, mais uma vez, tiveram um impacto significativo na sociedade brasileira. No Brasil, oficialmente, foi relatada a ocorrência de 795 desastres naturais, os quais causaram 1.094 óbitos e afetaram 12.535.401 pessoas. Quanto aos municípios, 2.370 foram afetados, sendo que $65,44 \%$ foi por eventos hidrológicos (Tabela 4). Outro aspecto relevante a ser observado, com o propósito de se identificar os períodos e regiões mais críticos para cada tipo de desastre, é a distribuição dos danos humanos. Apesar de a Região Sul ter sido a mais afetada pelos desastres em 2011 (6.855.449 afetados), a que sofreu o maior impacto pelo poder de destruição deles foi a Sudeste. A quantidade de óbitos verificada nesta região é 7,29 vezes maior do que a verificada nas outras quatro juntas, muito em razão do evento ocorrido na Região Serrana do Rio de Janeiro. Isso representa $87,95 \%$ do total de óbitos ocasionados por desastres no Brasil em 2011.
Minas Gerais tem registrado elevado número de municípios que são afetados pelas chuvas, devido, principalmente, a desastres relacionados com inundações e deslizamentos. A Tabela 5 contêm os dados de registros desde o ano de 2005.

Tabela 4-Quantidade de Municípios Afetados nas Macrorregiões do Brasil - 2011. (Fonte: Defesa Civil, 2013)

\begin{tabular}{lc}
\hline Região & $\begin{array}{c}\text { Quantidade de municípios afetados } \\
(\mathbf{2 3 7 0})\end{array}$ \\
\hline Norte & 77 \\
\hline Nordeste & 355 \\
\hline Centro-Oeste & 122 \\
\hline Sudeste & 569 \\
\hline Sul & 1247 \\
\hline
\end{tabular}

Tabela 5 - Balanço dos danos sofridos em municípios de Minas Gerais durante as chuvas para os anos de 2005 a 2012/201 (Fonte dos dados: Defesa Civil/MG)

\begin{tabular}{|c|c|c|c|c|c|c|c|c|}
\hline Balanço das chuvas & $2005 / 2006$ & $2006 / 2007$ & $2007 / 2008$ & $2008 / 2009$ & $2009 / 2010$ & $2010 / 2011$ & $2011 / 2012$ & $2012 / 2013$ \\
\hline Municípios Afetados & 152 & 316 & 121 & 276 & 120 & 216 & 273 & 88 \\
\hline \multicolumn{9}{|c|}{ Municípios que Decretaram Situação de Anormalidade } \\
\hline $\begin{array}{c}\text { Situação de emergência } \\
\text { (SE) }\end{array}$ & 73 & 238 & 49 & 218 & 69 & 167 & 239 & 43 \\
\hline \multicolumn{9}{|c|}{ Danos Humanos } \\
\hline Óbitos & 16 & 26 & 20 & 44 & 20 & 23 & 20 & 24 \\
\hline Feridos & 153 & 301 & 45 & 483 & 68 & 439 & 346 & 13 \\
\hline Desabrigados & 6100 & 14771 & 4354 & 11630 & 2406 & 4303 & 9594 & 0 \\
\hline Desalojados & 14600 & 54331 & 1875 & 113548 & 11206 & 37876 & 106618 & 7180 \\
\hline Afetados & 171758 & 699311 & 72979 & 1174958 & 861083 & 15686 & 3624274 & 943 \\
\hline \multicolumn{9}{|c|}{ Danos Materiais } \\
\hline Casas destruídas & 1211 & 1521 & 94 & 1100 & 169 & 352 & 1436 & 55 \\
\hline Casas danificadas & 6050 & 9568 & 2101 & 29523 & 4958 & 7858 & 23821 & 743 \\
\hline Pontes destruídas & 212 & 710 & 24 & 419 & 122 & 371 & 886 & 79 \\
\hline
\end{tabular}


Em Belo Horizonte, capital de Minas Gerais, durante os períodos chuvosos de 2009 até 2012 foram registrados desabamentos totais e parciais de edifícios e casas, inundações e enchentes, inclusive em áreas de elevado padrão construtivo (Figuras 2 a 5).

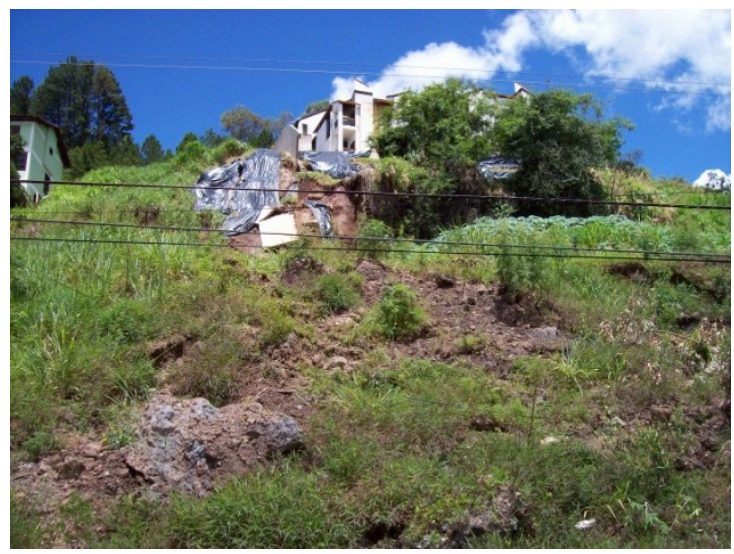

Figura 2 - Escorregamento em talude do bairro Mangabeiras, Belo Horizonte. A casa no topo está com estrutura abalada. Foto de 2012

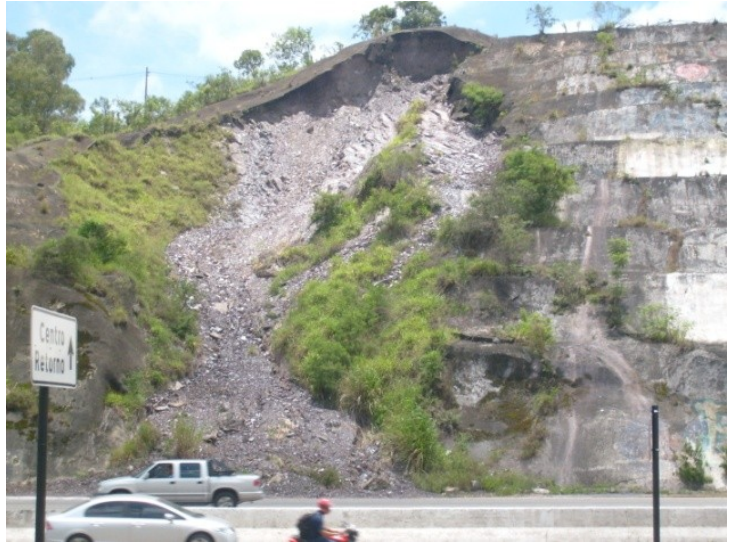

Figura 3 - Escorregamento em talude da BRO4O criando situação de perigo. Foto de 2011

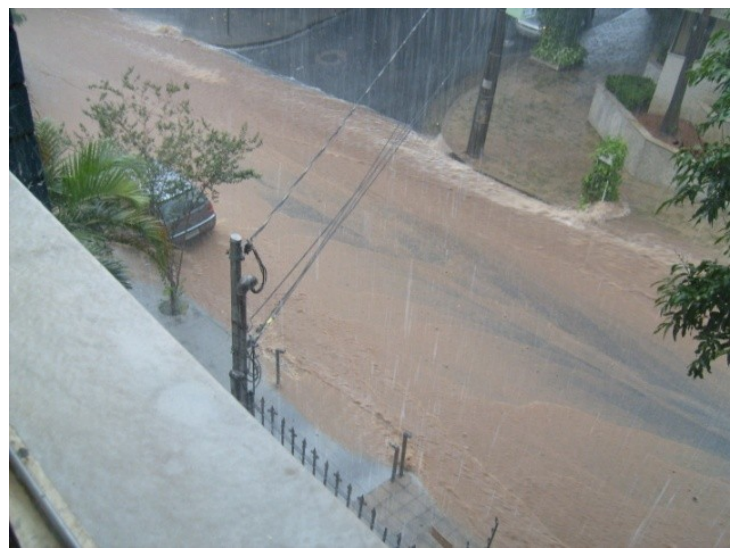

Figura 4 - Enxurrada brusca em rua declivosa no bairro Anchieta em Belo Horizonte. Foto de 2009

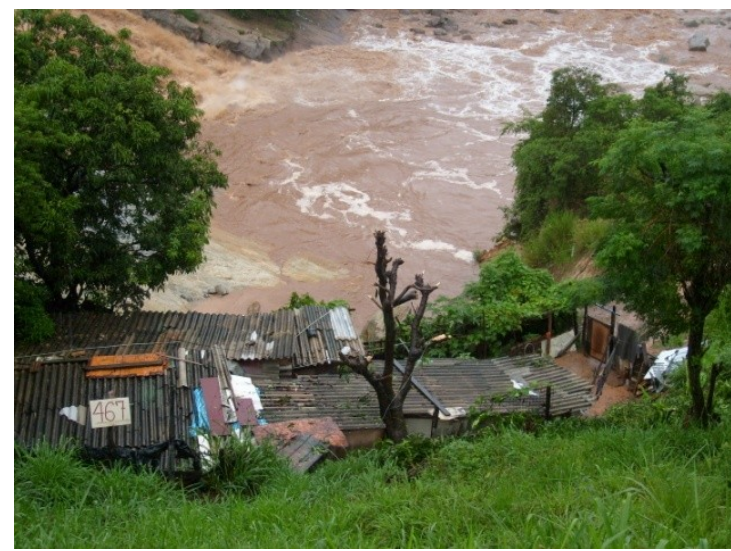

Figura 5 - Enchente do ribeirão Arrudas, Belo Horizonte. Foto de 2009.

\section{PROCESSOS E VULNERABILIDADE}

A ocorrência e a intensidade dos desastres dependem, em grande parte, do grau de vulnerabilidade dos cenários de desastres e das comunidades afetadas, e não somente da magnitude dos eventos adversos. Assim, por exemplo, terremotos com magnitude de 6.5 graus na escala Richter provocaram as seguintes perdas humanas: cinco óbitos na Califórnia; 20 mil óbitos, no Cairo; 40 mil óbitos, na Armênia. (Ministério da Integração Nacional, 2007)

No Brasil ocorre, com frequência, a ocupação desordenada do solo em áreas não edificáveis ocasionando um aumento na vulnerabilidade às enchentes, enxurradas, alagamentos e outros processos. Uma mesma quantidade de chuva em municípios diferentes pode induzir danos humanos, ambientais e materiais completamente diferentes, em função, especificamente, da vulnerabilidade. 0 intenso processo de urbanização no Brasil nas últimas décadas associado à falta de recursos e de políticas públicas permanentes que priorizem estes problemas, tem mantido uma situação social que força a população de mais baixa renda a ocupar áreas geologicamente desfavoráveis. Geralmente formam-se as chamadas favelas, onde normalmente são encontradas graves situações de risco.

A correlação entre pobreza e risco fica mais clara à medida que aumentam as informações sobre a ocorrência de desastres. Evidências de todas as regiões do mundo mostram que catástrofes produzem declínio no rendimento, consumo e indicadores de desenvolvimento humano e que estes efeitos desproporcionalmente estão concentrados nas comunidades e famílias pobres (Jha et al., 2010). Os efeitos de catástrofes são especialmente pronunciados em alguns dos indicadores de desenvolvimento humano, a maioria importante 
para a redução da pobreza: produtividade, saúde e educação. Famílias pobres têm uma capacidade limitada para recuperar-se contra perdas de desastres em locais onde os riscos são intensos ou extensos. Eles também podem ter proteção social limitada, dependendo em grande parte das medidas públicas disponíveis durante a recuperação de desastres (Jha et al., 2010).

Para a América Latina, Maskrey (1992) considera que várias condições de alta vulnerabilidade são geradas e reproduzidas nos processos de desenvolvimento regionais e de centros urbanos. Estas condições de vulnerabilidade são principalmente:

- Aumento brutal da população em áreas urbanas sujeitas a acidentes;

- Uma das consequências dos acidentes é a interrupção das atividades econômicas, levando ao desemprego da população;

- Falta de capacidade econômica para suportar os prejuízos;

- Incapacidade da população para proteger a si própria, vivendo em locais inadequados, com moradias de baixa qualidade, etc.;

- Inexistência de proteção dada pela sociedade, como, por exemplo, auxílio médico; e

- Falta de conhecimentos e informações acerca dos riscos, principalmente quando os acidentes são escassos no tempo.

\section{GESTÃO DO RISCO EM ÁREAS URBANAS}

A gestão do risco em áreas urbanas é um processo social complexo que envolve ações de planejamento, intervenção e organização, que devem ser avaliadas e conduzidas de forma contínua e consistente em cada fase do desastre (Lavell, 2003). Tobin \& Montz (1997, apud Marcelino 2008) estabelecem que a ocorrência de desastres envolve basicamente três fases distintas: Antes, Durante e Depois.

O Antes se refere à fase que antecede a chegada dos fenômenos extremos, conhecida como etapas de Prevenção e Preparação para o impacto. Considerada a fase mais importante em que são adotadas medidas para reduzir o impacto dos desastres, as análises de risco, a execução de projetos de engenharia (diques, pontes, muros de contenção, etc.), a elaboração de políticas públicas (plano diretor, zoneamentos ambientais, legislação, etc.), a educação ambiental em escolas e comunidades afetadas, etc. Nos momentos mais críticos que precedem o impacto, destacam-se os sistemas de previsão (meteorológica e hidrológica) e de alerta. Quanto maior for o investimento nesta fase, menor será a probabilidade de danos, podendo até mesmo acarretar na diminuição da frequência e intensidade dos desastres.

O Durante envolve a realização de ações emergenciais (fase de RESPOSTA) que visam o salvamento (socorro e assistência às vítimas), o auxílio (evacuação, abrigo, alimentação, atendimento médico, etc.) e a reabilitação do cenário do desastre, que corresponde ao início das atividades de restauração (curto prazo), como limpeza, segurança, entre outros.

O Depois (fase de Reconstrução) é representado pelas atividades de restabelecimento das funções básicas de uma comunidade (bem-estar da população) a médio e longo prazo. É realizado o restabelecimento dos serviços essenciais (água, luz, comunicação e transporte), a avaliação dos danos e, nas áreas mais afetadas, a reconstrução das estruturas danificadas ou destruídas (casas, pontes, estradas, etc.). O objetivo na fase de Reconstrução, ampliado pela fase de Prevenção, é retornar à normalidade, entendida como sinônimo de funcionalidade, e não de retorno às condições que precederam o desastre. $O$ objetivo é fazer que a área afetada retorne às suas funções socioeconômicas primordiais, mas de forma compatível com a realidade ambiental que a cerca, o que resultaria numa elevação da qualidade de vida local. Dessa forma, a vulnerabilidade e o risco de desastres tenderiam a diminuir de forma natural.

Em 1991, a UNDRO (Disaster Management Training Programme) definiu um modelo de abordagem do risco estruturado em cinco etapas:

- 1. Identificação do risco

- 2. Análise do risco

- 3. Medidas de prevenção de acidentes

- 4. Planejamento para situações de emergência

\section{- 5. Informações públicas e treinamento}

De acordo com o Ministério das Cidades \& IPT (2004) as duas primeiras etapas acima devem ser fundamentadas na Previsão, ou seja, possibilitar a identificação das áreas de risco com a indicação dos locais onde poderão ocorrer acidentes (definição espacial $=$ ONDE) e qual o tipo de processo envolvido (= O QUE e COMO). Também possibilitam o estabelecimento das condições e circunstâncias para a ocorrência dos processos (definição temporal = QUANDO).

As etapas seguintes pertencem à fase de prevenção. Abordam a possibilidade de serem 
adotadas medidas preventivas visando, ou inibir a ocorrência dos processos, ou reduzir suas magnitudes, ou ainda minimizar seus impactos, agindo diretamente sobre edificações e/ou a própria população (O QUE FAZER).

Marcelino (2008) ressalta que a identificação e avaliação de risco é um dos principais passos que vai nortear as demais etapas do processo de gestão. A avaliação de risco, envolve basicamente o inventário dos perigos naturais $(\mathbf{P})$, o estudo da vulnerabilidade (V) e o mapeamento das áreas de risco $(\mathbf{R})$, sendo

$$
\mathbf{R}=\mathbf{P} \times \mathbf{V}
$$

Esses parâmetros podem ser cruzados facilmente em ambiente SIG (Sistema de Informação Geográfica) para obtenção do mapa de risco final. Cada parâmetro é formado por um conjunto de dados de fontes diversas (mapas, medições em campo, imagens de satélites, questionários, etc.), que permitem identificar as características do ambiente e o contexto socioeconômico em que podem ocorrer os desastres. Devido à grande complexidade de variáveis e tipos de dados, assim como à forma de mensuração, as avaliações de risco geralmente envolvem muita incerteza. De forma simplificada, os principais dados requeridos numa avaliação de risco são representados na tabela 6 .

Tabela 6: dados usados numa avaliação de risco (Marcelino,2008)

\begin{tabular}{ll}
$\begin{array}{l}\text { Dados sobre o } \\
\text { perigo }\end{array}$ & $\begin{array}{l}\text { Tipo de processo, data, local de ocorrência, } \\
\text { freqüência, magnitude, etc. }\end{array}$ \\
\hline $\begin{array}{l}\text { Dados sobre o } \\
\text { ambiente }\end{array}$ & $\begin{array}{l}\text { Geologia, geomorfologia, hidrologia, } \\
\text { climatologia, uso da terra, etc. }\end{array}$ \\
\hline $\begin{array}{l}\text { Dados sobre a } \\
\text { exposição local }\end{array}$ & $\begin{array}{l}\text { Infra-estrutura urbana, edificações, } \\
\text { população, dados sócio-econômicos, } \\
\text { agropecuários, etc. }\end{array}$
\end{tabular}

Nogueira (2002) incluiu o grau de gerenciamento do risco passível de ser implantado em uma área urbana como parâmetro quantificador do risco de escorregamentos, de acordo com a formulação:

$$
R=P(f A) * C(f V) * g^{-1}
$$

onde um determinado nível de risco $\mathbf{R}$ representa a convolução da possibilidade ou probabilidade $\mathbf{P}$ de ocorrer um fenômeno físico $\mathbf{A}$, em local e intervalo de tempo específico e com características determinadas (localização, dimensões, processos e materiais envolvidos, velocidade e trajetória), causando conseqüências $\mathbf{C}$ (às pessoas, bens e/ou ao meio ambiente), em função da vulnerabilidade $\mathbf{V}$ (indicativa da suscetibilidade e do nível de resiliência dos elementos expostos), podendo ser modificado pelo grau de gerenciamento $\mathbf{g}$, existente ou passível de ser implantado.

Cerri \& Amaral (1998) enfatizam que a distribuição do risco geológico pode ser representada de duas maneiras na chamada Carta de Risco:

- cadastramento de risco, no qual são plotados os pontos notáveis sujeitos a risco, com indicação do grau de risco, acompanhado da adequada documentação sobre processos geológicos e possíveis danos, representados em croquis esquemáticos e fotografias locais;

- Zoneamento de risco, no qual são delimitadas as zonas homogêneas em relação ao grau de risco geológico, estabelecendo tantas classes de áreas de risco quantas necessárias, levando-se em consideração a quantidade e a qualidade das informações coletadas.

Em geral as cartas de risco contam com quatro domínios de risco: RISCO ALTO, RISCO MODERADO, RISCO BAIXO E RISCO INEXISTENTE. No caso da elaboração de cartas de risco em áreas afetadas por mais de um processo geológico causador de risco, deve-se estabelecer legendas e zonas de risco discriminadas para cada um dos processos. A carta de risco, acompanhada do relatório técnico, deve contar com um mapa que indique todas as situações de risco naturais identificadas. Uma das funções da carta é permitir a visita aos locais em risco por técnicos para a definição de projetos de solução localizada.

A formulação e a proposição de medidas de prevenção de acidentes devem ser efetuadas logo em seguida à elaboração das cartas de risco (indicação dos locais ameaçados, quantificação e estabelecimento de prioridades).

A prevenção de acidentes deve considerar os seguintes objetivos:

- eliminar e /ou reduzir os riscos já instalados;

- evitar a instalação de novas áreas de risco;

- conviver com os riscos atuais.

Eliminar o risco pressupõe a remoção definitiva da população da área, o que nas condições de carência de áreas adequadas para receber a população e de recursos financeiros suficientes, não é comum se observar.

A redução dos riscos pode se dar pela implantação de obras de engenharia.

A convivência em níveis relativamente seguros se dá por meio da operação de sistemas de alerta ou planos preventivos. 
Tais medidas consistem basicamente em manter continuamente as áreas sob controle, através de vistorias, e proceder a retirada dos moradores quando a situação se configurar como de alta possibilidade de deflagração de processos.

A seleção da medida preventiva mais indicada depende:

- do entendimento do(s) processo(s) geológicos(s) considerados(s);

- das dimensões das áreas de risco;

- da disponibilidade de recursos financeiros, tempo e áreas para a relocação da população;

- de uma decisão político-administrativa.

A tabela 7 reune as alternativas técnicas para o gerenciamento do risco, de acordo com Ministério das Cidades \& IPT (2004).

Como afirma Macedo (2001), embora haja uma preocupação com o risco, poucas Prefeituras Municipais têm efetivamente estabelecidos sistemas eficientes de gerenciamento de áreas de risco. Faltam a essas municipalidades, além de priorizar a questão, equipes técnicas com formação adequada para o atendimento desses problemas. Os poucos trabalhos em andamento nas prefeituras são frutos de equipes que contam com geólogos, engenheiros, arquitetos, geógrafos, assistentes sociais e outros profissionais, com boa formação na área, em alguns casos, e conhecimentos práticos, que possam ser usados emergencialmente, em outros. Assim, alia-se ao pequeno contingente de técnicos com formação adequada, a incapacidade das prefeituras em montar equipes e quando estas existem, a formação básica de muitos desses profissionais, impede que eles possam reproduzir as metodologias completas de gerenciamento de riscos geológicos.

É necessário, então, que se desenvolvam metodologias que possam ser repassadas para profissionais, não somente das áreas de geociências e engenharia. Este caminho poderá permitir a formação de maior número de técnicos municipais, de modo que possam atender às necessidades dos municípios com um grau de acerto, que pode não ser igual ao de um profissional especializado, mas que seja com um mínimo de segurança. A formação desses profissionais pode contar com a ajuda dos estudos de percepção ambiental ou de riscos, que utiliza os conhecimentos pré-existentes, buscando melhorar o desempenho dos profissionais sem se descuidar de suas experiências de vida.

Tabela 7: Alternativas técnicas para o gerenciamento do risco (IPT \& Ministério das Cidades, 2004)

MEDIDAS DE PREVENÇÃO DE ACIDENTES:

\section{ESTRUTURAIS}

- OBRAS, ex: Obras de contenção, drenagem, proteção superficial

- Reurbanização

- Relocação de moradias e população

\section{NÃO-ESTRUTURAIS}

- Planejamento urbano

- Cartas geotécnicas e de risco

- Planos Preventivos de Defesa Civil

- Legislação

- Educação e capacitação

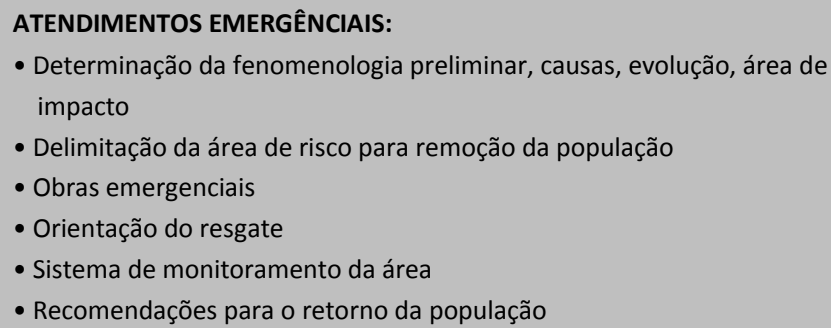

\section{INFORMAÇÕES PÚBLICAS E TREINAMENTO:}

- Cursos, palestras, seminários, reuniões

- Publicações técnicas

- Cartilhas, folders, cartazes

- Simulados 


\section{CONCLUSÃO}

O gerenciamento do risco deve ser uma ação prioritária e permanente nas cidades. Conforme relatado, os centros urbanos crescem cada vez mais e, muitas vezes, a forma de ocupação ou uso é inadequada ao tipo de terreno (geologia e geomorfologia). Isso aumenta a vulnerabilidade e, consequentemente, o grau de risco. Seja qual for o fenômeno causador do desastre (terremoto, furacão, vulcão, chuvas intensas, deslizamentos, etc.), os danos podem ser atenuados ou até inexistentes se a ocupação for realizada de modo racional, visando conhecer e respeitar o equilíbrio, a dinâmica natural e as características físicas do ambiente a ser ocupado. Várias entidades mundiais de monitoramento de desastres afirmam (CRED; IPCC- Intergovernmental Panel on Climate Change; ISDR, UNDRO) que indivíduos e comunidades são diferencialmente vulneráveis baseado em desigualdades expressas pelos níveis de riqueza e educação, capacidade e condições de saúde, bem como gênero, idade, classe e outras características sociais e culturais. Padrões de assentamento, urbanização e alterações nas condições socioeconômicas têm influenciado tendências observadas em exposição e vulnerabilidade a desastres. A redução da pobreza e melhoria das condições de saneamento e infraestrutura são aspectos que interferem na redução dos impactos relativos a desastres e na resiliência das comunidades. Muitas das preocupações com desastres em todo o mundo poderiam concentrar esforços em campanhas de conscientização da necessidade da ocupação adequada, discutindo a questão da OCUPAÇÃO GLOBAL e da REDUÇÃO DA POBREZA, e, assim, reduzir a vulnerabilidade das cidades.

\section{REFERÊNCIAS}

BERTRAND, G. Paisagem e geografia física global: esboço metodológico. Tradução Olga Cruz - Caderno de Ciências da Terra. Instituto de Geografia da Universidade de São Paulo, no13, 1972, P.1-27.

Cerri, L.E. da S. \& Amaral, C. Riscos Geológicos. 1998. In: Oliveira, A.M dos S., Brito, S.N. (organizadores), Geologia de Engenharia. São Paulo, ABGE (Associação Brasileira de Geologia de Engenharia), 1998, P.301-310.

CRED-EM-DAT - - Annual Disaster Statistical Review 2008 - The numbers and trends. Org. Rodriguez, J.; Vos, F.; Below, R.; Guha-Sapir, D. Centre for Research on the Epidemiology of Disasters. Jacoffset Printers, Melin (Belgium) 2009. 33p.

Centre for Research on the Epidemiology of Diseases (CRED). 2011. Université Catholique de Louvain, Ecole de Santé Publique. Brussels. http://www.cred.be/. Acesso em nov. 2011.

CRED-EM-DAT - Emergency Events Database. The OFDA/CRED, 2011 International Disaster Database. Disponível em: http://www.em-dat.net/. Acesso em: 02 Nov. 2012.
CRED-EM-DAT - “Disaster Data: A Balanced Perspective” - 2013. Issue No. 31, March 2013. Disponível em: http://www.emdat.net/. Acesso em: 31 Jul. 2013.

Defesa Civil, Minas Gerais. 2013. Boletim Estadual de Defesa Civil. Disponível http://www.defesacivil.mg.gov.br/index.php/manuaisapostilas. 2013. 10p. Acesso em Jul. de 2013.

Jha, A.K, Barenstein, J.D; Phelps, P.M.;Pittet, D.; Sena, S.Safer 2010. Homes, Stronger Communities. A Handbook for Reconstructing after Natural Disasters. The World Bank (The International Bank for Reconstruction and Development), GFDRR (Global facility For Disaster Reduction And Recovery). 2010. 407p

Lavell, A. 2003. La gestión local del riesgo: nociones y precisiones en torno al concepto y la práctica. Guatemala: CEPREDENAC/PNUD, 2003. $101 \mathrm{p}$.

Macedo, E. S. 2001. Elaboração de cadastro de risco iminente relacionado a escorregamentos: avaliação considerando experiência profissional, formação acadêmica e subjetividade. Tese (Doutorado em Geociências e Meio Ambiente) - Instituto de Geociências e Ciências Exatas, Universidade Estadual Paulista.Rio Claro, 2001, 276 p.

Marcelino, E. V. Desastres Naturais e Geotecnologias: Conceitos Básicos. 2008. Caderno Didático no 1. INPE/CRS, Santa Maria, 2008.38p.

Maskrey, A. 1992. Introducción. In: Medina, j., Romero, R. (Eds.). Los desastres si avisan: estudios de vulnerabilidad y mitigación. Lima: ITDG. p.13-25.

Ministério das Cidades - Brasil /Instituto de Pesquisas Tecnologicas/IPT. 2004. Curso:Treinamento de Técnicos Municipais para o Mapeamento e Gerenciamento de Áreas Urbanas com Risco de Escorregamentos, Enchentes e Inundações. Carvalho,C.S., Macedo, E.S. e Ogura, A.T, Organizadores - Brasilia: Ministério das Cidades; Instituto de Pesquisas Tecnologicas - IPT, 2004.73p.

MMA - Ministério do Meio Ambiente. 2007. Vulnerabilidade Ambiental. Desastres naturais ou fenômenos induzidos? Santos, Rosely F. (org). Brasília: MMA 2007.192 p.

Ministério da Integração Nacional, BRASIL. 2003. Manual de desastres humanos: desastres humanos de natureza tecnológica (Vol. 1a Parte da Natureza tecnológica). (M. d. Civil, Ed.) Brasília: Ministério da Integração Nacional. Secretaria Nacional de Defesa Civil, 2003.

Ministério da Integração Nacional, Brasil. 2007. Segurança global da população. Brasília, Secretaria Nacional de Defesa Civil. 2007, 65p.

Nogueira, F.R. 2002. Políticas públicas municipais para gerenciamento de riscos ambientais associados a escorregamentos em áreas de ocupação subnormal. Tese (Doutorado em Geociências e Meio Ambiente) Universidade Estadual Paulista. Rio Claro. 2002. 256p.

UN-ISDR - International Strategy for Disaster Reduction. 2011. Terminology on Disaster Risk Reduction. 2009. Disponível em http://www.unisdr.org. Acesso em Jul. de 2013.

UN-ISDR - International Strategy for Disaster Reduction. 2011 Evidence. Disponível em http://www.unisdr.org. Acesso em Jul. de 2013.

UNDRO - United Nations Disaster Relief Office. UNDRO's approach to disaster mitigation. UNDRO News, jan.febr.1991. Geneva: Office of the United Nations Disasters Relief Co-ordinator. 20p. 